\title{
Contamination of Toxic Volatile and Semi-Volatile Compounds from Automobile Materials
}

\author{
S. Zhou, ${ }^{1,3}$ J. X. Huang, ${ }^{1}$ J. Kinkel, ${ }^{2}$ W. Stark ${ }^{2}$ \\ ${ }^{1}$ State Key Laboratory of Environmental Chemistry and Ecotoxicology, Research \\ Center for Eco-Environmental Sciences, Chinese Academy of Sciences, Graduate \\ School of Chinese Academy of Sciences, Beijing, 100085, People's Republic of China \\ ${ }^{2}$ Technical Chemistry Department, University of Applied Sciences, George Simon \\ Ohm Fachhochschule Nurnberg, Kesslerplatz 12, 90489, Nurnberg, Germany \\ ${ }^{3}$ Department of Central Laboratory, Beijing Municipal Center for Disease Prevention \\ and Control, Number 16, Hepinglizhongjie, Dongcheng District, Beijing, 100013, \\ People's Republic of China
}

Received: 5 July 2005/Accepted: 15 November 2005

Automobile industry has been rapidly developed and pollution it may bring to the environment has been a big concern. For decades, focus has been put on the fuels that autos are using and alternative fuels have been investigated. Researchers have also concerned about materials used in automobiles that may contain detrimental compounds and bring in potential risks to people's health (Elke et al. 1998; Guadarrama et al. 2002). Volatile or semi-volatile toxic substances are more likely to release to either the cabins of cars or straight to the atmosphere. Odors as well as toxic compounds in the cabins of cars and outer layer, may have adverse effects to drivers and passengers, even more, when releasing to the outdoor atmosphere, they will affect more people at a much larger scale. Materials used in cars can fall into varieties of categories of different chemical compounds. As a consequence, to monitor compounds released from automobile materials is a challenge for analytical chemists given that sample matrix can be extremely complicated and level of compounds involved can be very low. Therefore, it requires an analytical method to be both sensitive and as less influenced by the matrix as possible.

Conventional techniques to determine organic compounds involve several processes among which sampling and sample pretreatment are very critical steps. In recent years, a technique SPME has been extensively applied as a sample pretreatment method, the major advantage of this new technology is that it combines sampling, extraction, concentration and injection into one process, it is fast and easy to operate, above all, it is solvent free and has been regarded as an overall environmental friendly technique (Beltran et al. 2000; Kataoka et al. 2000; Lambropoulou et al. 2003). By coupling SPME with GC-MSD, it allows us to extract and concentrate samples before they are injected into GC port, and thus makes it possible to detect more compounds even at trace concentrations (as low as sub ppb levels). There are several SPME fibers that are commercially available and can therefore be employed for researchers. PDMS fiber is mainly designed for VOCs, however CAR-PDMS fiber has more affinity to polar compounds at lower molecular mass. Both of these two types of fibers were applied in our study and possibility of detecting more compounds was expanded. The objective of this study was to establish a fast and effective method to do rapid screening of VOCs and semi-VOCs from materials used in automobiles and to get a profile of toxic substances present in those materials.

\section{MATERIALS AND METHODS}

Five types of materials from different parts of trucks were collected from a local automobile company in Nurnberg, Germany. Samples were cut into small pieces,

$\overline{\text { Correspondence to: }}$ S. Zhou at affiliation 3 
weighed and filled into $15 \mathrm{~mL}$ HS sample vials, occupying about half of the total volume. 3 replicates of each material were determined following the operation procedure described below. $100 \mu \mathrm{m}$ PDMS and $85 \mu \mathrm{m}$ CAR-PDMS fibers were applied in this study, HS system was used and vials that contain samples were equilibrated for 10minutes, then SPME fibers were exposed at the HS portion of sealed sample vials at $50^{\circ} \mathrm{C}$ for 15 minutes. After extraction, fibers were taken into the injection port and let desorb at $250^{\circ} \mathrm{C}$ for 10 minutes. A $30 \mathrm{~m} * 0.25 \mathrm{~mm} * 0.25 \mu \mathrm{m}$ HP-5 MS column was employed; GC oven temperature program was applied. Identification of compounds was preformed by NIST library search, as well as visual examination. Two sets of standards which were C5 to C20 alkanes and concentrated diesel were administrated with the same GC-MS program and theory of Kovats Retention Time system was applied to exclude unlikely compounds appeared in the library search report.

\section{RESULTS AND DISCUSSION}

Extraction time and temperature are both very critical parameters for SPME operation. At higher temperature, more compounds are expected to release from the sample medium and escape to the headspace, therefore more are extracted to the surface of fibers. However SPME is a thermo releasing process, so that lower temperature favors the extraction itself. As a consequence, it needs a "trade-off" optimization. As for extraction time, longer duration allows the compounds to reach their equilibrium among solid sample, headspace and extraction fiber surface, therefore, for the compounds that have more polarity, less volatility and more molecular mass, the longer the extraction time, the more sufficient the extraction reaches. Both extraction time and temperature are in fact compound dependant which means that VOCs with high volatility and low molecular mass need lower temperature and less time for extraction and vice versa. In our research, 10,15 and 30 minutes combined with 50,60 and $70{ }^{\circ} \mathrm{C}$ were tested individually. Medium extraction time and lower temperature seemed to favor our analysis, 50 ${ }^{\circ} \mathrm{C}$ was found to extract almost same amount of compounds in numbers (Table1) but with higher intensity (Fig 1), so that it was selected as the final operation temperature. Because VOCs and Semi-VOCs were dealed with, it doesn't need much time to reach equilibrium and 15 minutes provided both the biggest amount of compounds detected in numbers and the best sensitivity in terms of intensity for our study (Fig 2) so that 15 minute was chosen as extraction time duration.

Two types of fibers were employed in the study and except for sample 1, for all the other cases, $100 \mu \mathrm{m}$ PDMS fiber was found to be more efficient in terms of number of compounds detected (Table 1) and intensity of them. It is not surprising since this type of fiber has thicker coatings and is non-polar, for VOC or semi-VOC analysis, it has the most capacity, sensitivity and selectivity. However, the combination of two types of fibers allows wider range of compounds to be extracted and detected and this was confirmed by our study. Some more polar compounds at relatively lower molecular masses such as phenol, benzyl alcohol, benzoic acid, phthalic anhydride that were not successfully detected by $100 \mu \mathrm{m}$ PDMS fibers were detected by $85 \mu \mathrm{m}$ CAR-PDMS fiber.

Five materials from different parts of automobiles were examined and from three of the five materials (Samples 1 to 3), between 30 and 60 VOCs and semi-VOCs were detected. Many of these compounds are styrene or phenyl compounds known to be detrimental. These three materials were used as fire retardant layers, curtains and flooring of the driving compartments of trucks. In addition to styrene and phenyl compounds, ketones, carboxylic acids, esters, alkynes, phenols, 
phthalates, naphthalene alcohols and aldehydes were detected. Azulene was detected in Sample 2 and alkenes in Sample 3. Samples 4 and 5 were isolation and panel materials and these yielded fewer compounds but included amines. Major components detected were listed in Table 2. Since there was considerable amount of toxic VOCs and Semi-VOCs detected in materials of automobiles, it warned that selection of materials in cars should be more careful and more cautions should be taken in material processing, additives using and so on. When looking into environmental issues that automobiles may generate to the surroundings, not only fuels, but also materials used in different parts of automobiles could release toxic compounds to cabins of cars or outdoor air so that it may threaten health of drivers, passengers, as well as other people in a larger scale.

Table 1. Number of compounds detected with different extraction parameters.

\begin{tabular}{lllllll}
\hline & $50^{\circ} \mathrm{C}$ & $60^{\circ} \mathrm{C}$ & $70^{\circ} \mathrm{C}$ & $50^{\circ} \mathrm{C}$ & $50^{\circ} \mathrm{C}$ & $50^{\circ} \mathrm{C}$ \\
\hline & $15 \mathrm{~min}$ & $15 \mathrm{~min}$ & $15 \mathrm{~min}$ & $10 \mathrm{~min}$ & $15 \mathrm{~min}$ & $30 \mathrm{~min}$ \\
PDMS & $1^{\#}$ & $1^{\#}$ & $1^{\#}$ & $2^{\#}$ & $2^{\#}$ & $2^{\sharp}$ \\
CAR-PDMS & 54 & 47 & 47 & 26 & 40 & 36 \\
\hline
\end{tabular}

Table 2. Major components in 5 different materials.

\begin{tabular}{l|ll}
\hline & Compound & Peak area percentage (\%) \\
\hline Spl 1 & Benzene, 1,3-dimethyl- & 12.3 \\
& Styrene & 31.2 \\
& Benzene, 1,2,3-trimethyl- & 23.6 \\
& Benzene, 1,3,5-trimethyl- & 12.9 \\
& Benzene, 1,2,4-trimethyl- & 3.6 \\
Spl 2 & Nonanal & \\
& Decanal & 22.0 \\
& Esters & 10.2 \\
& 1,1'biphenyl, 3,4-diethyl- & 10.3 \\
Spl 3 & & 4.8 \\
& butylated hydroxtoluene & \\
& Phenols & 11.8 \\
& Alcohols & 11.2 \\
& Alkenes & 11.5 \\
& & 30.2 \\
Spl 4 & Styrene & 18.1 \\
& Benzene, 1-ethyl-4-methyl- & 2.8 \\
& Phenol & 3.2 \\
& 4H-1, 3-benzodioxin & 4.3 \\
& & \\
& triethylenediamine & 27.7 \\
& Ethanol, 2-(2-butoxyethoxy)-, acetate & 6.0 \\
& 2,5-cyclohexadiene-1, 4-dione, 2,6-bis & 2.5 \\
& Propanoic acid, 2-methyl-, 2,2-dimethyl- & 5.0 \\
\hline
\end{tabular}




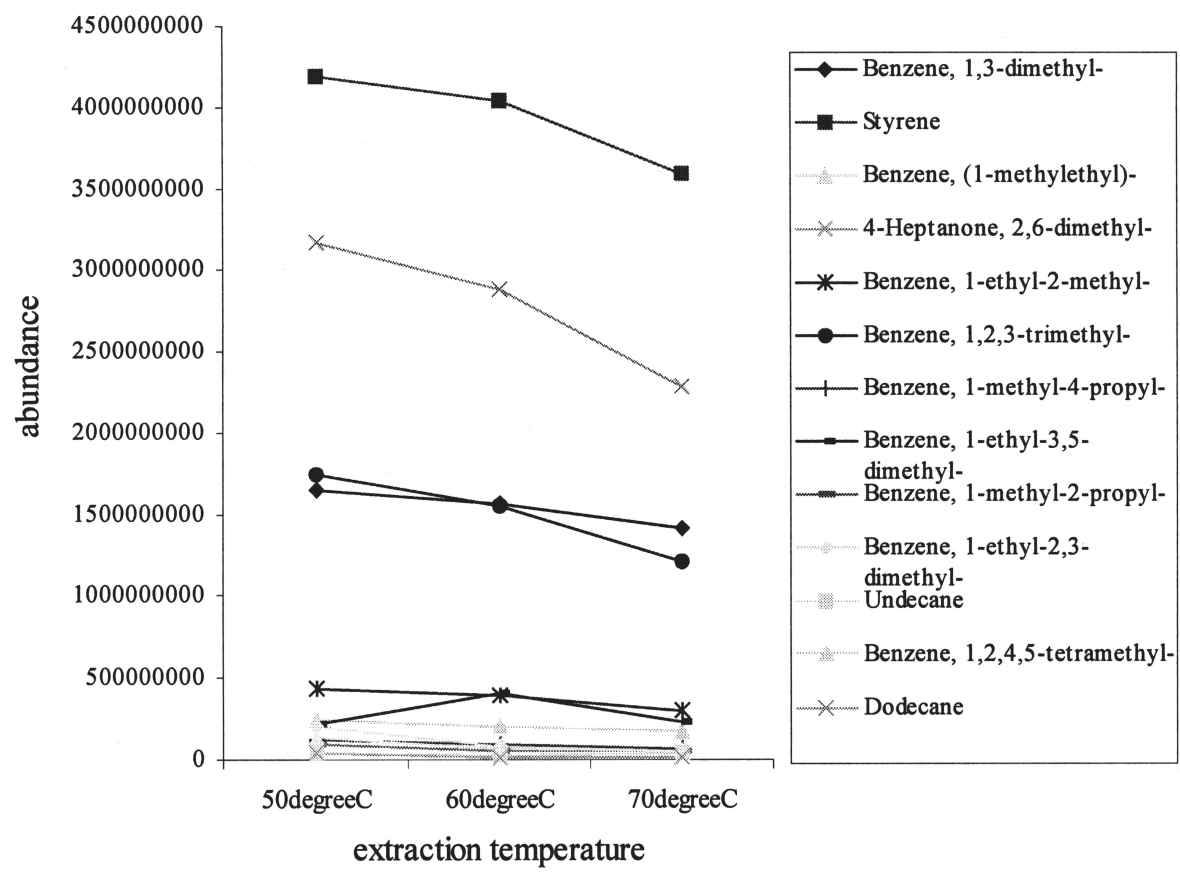

Figure 1. extraction temerature vs. abundance

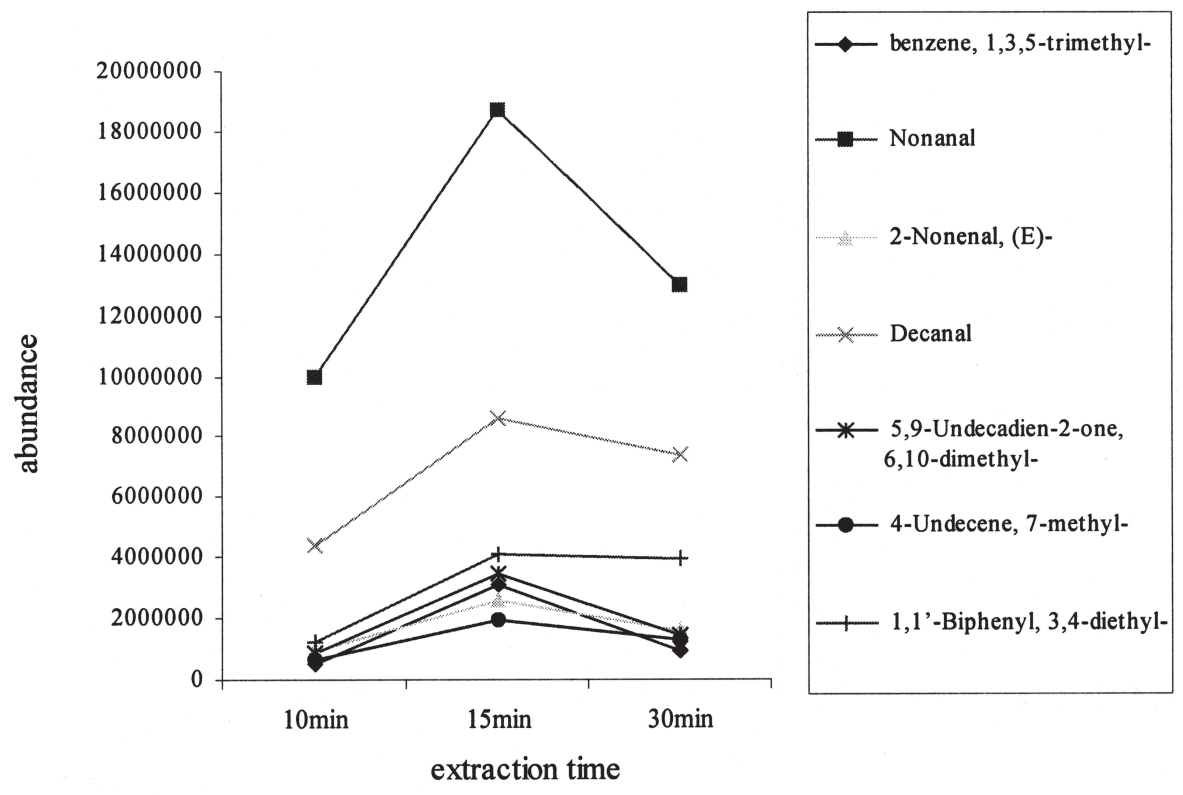

Figure 2. extraction time duration vs. abundance 
Acknowledgment. Sincere acknowledgment is given to Dr. Lothar Winkler and Mr. Bernhard Dusold in "MAN" Truck Company in Nurnberg, Germany for their providing samples. Thanks are also given to Mrs. Christine Muenzel, Mr. Christian Ulbricht, Miss. Kristin Rueffer and Mr. Thomas Billmann for their helping solving experimental problems.

\section{REFERENCES}

Beltran J, Lopez FJ, Hernandez F (2000) Solid-phase microextraction in pesticide residue analysis. J Chromatog A 885:389-404.

Elke K, Jermann E, Begerow J, Dunemann L (1998) Determination of benzene, toluene, ethylbenzene and xylenes in indoor air at environmental levels using diffusive samplers in combination with headspace solid-phase microextraction and high-resolution gas chromatography-fame ionization detection. J Chromatog A 826: 191-200.

Guadarrama A, Rodriguez-Mendez ML, de Saja JA (2002) Conducting polymer-based array for the discrimination of odors from trim plastic materials used in automobiles. Anal Chim Acta 455: 141-47.

Kataoka H, Lord HL, Pawliszyn J (2000) Applications of solid-phase microextraction in food analysis. J Chromatog A 880: 35-62.

Lambropoulou DA, Sakkas VA, Albanis TA (2003) Determination of antifouling compounds in marine sediments by solid-phase microextraction coupled to gas chromatography-mass spectrometry. J Chromatog A 1010: 1-8. 